\title{
Saikosaponin D Ameliorates Mechanical Hypersensitivity in Animal Models of Neuropathic Pain
}

(요 $(9 \Theta$

Authors

Gyeongbeen Lee ${ }^{1}$, Yeon-Ju Nam¹, Woo Jung Kim¹, Bo Hye Shin², Jong Suk Lee', Hwan Tae Park², 3, Pansoo Kim¹, ji Hyun Lee ${ }^{4}$, Yongmun Choi ${ }^{1}$

\section{Affiliations}

1 Biocenter, Gyeonggido Business and Science Accelerator, Suwon, Korea

2 Peripheral Neuropathy Research Center, Dong-A University College of Medicine, Busan, Korea

3 Department of Molecular Neuroscience, Dong-A University College of Medicine, Busan, Korea

4 Division of Hemato-oncology, Department of Internal Medicine, Dong-A University College of Medicine, Busan, Korea

Key words

Saikosaponin D neuropathic pain diabetes chemotherapy

received 09.06 .2020

revised 21.10.2020

accepted 29.10.2020

\section{Bibliography}

Planta Med Int Open 2020; 7: e145-e149

DOI 10.1055/a-1302-4570

ISSN 2509-9264

(c) 2020. The Author(s).

This is an open access article published by Thieme under the terms of the Creative Commons Attribution-NonDerivative-NonCommercial-License, permitting copying and reproduction so long as the original work is given appropriate credit. Contents may not be used for commercial purposes, or adapted, remixed, transformed or built upon. (https://creativecommons. org/licenses/by-nc-nd/4.0/)

Georg Thieme Verlag KG, Rüdigerstraße 14, 70469 Stuttgart, Germany

\section{Correspondence}

Dr. Yongmun Choi

Biocenter

Gyeonggido Business and Science Accelerator

107 Gwanggyro

Yeongtong-gu

Suwon 16229

Korea

Tel.: + 8231888 6972, Fax: + 82318886979

ychoi@gbsa.or.kr
Ji Hyun Lee, MD, PhD

Division of Hemato-oncology

Department of Internal Medicine

Dong-A University College of Medicine

26 Daeshingongwonro

Seo-gu

Busan 49201

Korea

Tel.: + 8251240 2915, Fax: + 82512402088

hidrleejh@dau.ac.kr

\section{ABSTRACT}

We have previously identified saikosaponins as transient receptor potential ankyrin 1 antagonists and showed that saikosaponin $D$ improves neuropathic pain induced by the anticancer drug vincristine in mice. In order to gain more insight into the therapeutic effects of saikosaponin D, we tested saikosaponin $D$ in animal models of neuropathic pain induced by either streptozotocin, which mimics diabetes, or paclitaxel, a commonly used chemotherapy treatment. Our findings indicate that although saikosaponin D improved pain outcomes in neuropathic pain models, the mechanisms underlying the therapeutic effects of saikosaponin D appear to differ between streptozotocin- and paclitaxel-induced pain. Streptozotocininduced neuropathic pain was significantly alleviated 30 minutes after oral administration of saikosaponin D, while 1-day oral administration of saikosaponin $\mathrm{D}$ had little effect on paclitaxel-induced mechanical hypersensitivity. Attenuation of paclitaxel-induced mechanical hypersensitivity was evident only after repeated administration of saikosaponin D. Although the mechanisms underlying the therapeutic effects of saikosaponin $D$ remain to be elucidated, our results shed new light on the therapeutic potential of saikosaponin D in the management of neuropathic pain caused by diabetes or chemotherapy. 


\section{Introduction}

The clinical manifestation of neuropathic pain is characterized by either spontaneous pain that comes without stimulation, an increased sensitivity to noxious stimuli, or abnormal sensitivity to otherwise innocuous stimuli (allodynia) [1,2]. The mechanism for neuropathic pain involves maladaptive changes in neural circuits after injury to the peripheral nervous system and central nervous system $[3,4]$. Although the exact etiology of neuropathic pain remains unclear, nerve damage is caused by many conditions, including diabetes, chemotherapy, nerve compression, viral infection, and tumor invasion [5].

In animal models of neuropathic pain induced by diabetes, anticancer drugs, and chronic constriction injury (CCI), the transient receptor potential ankyrin 1 (TRPA1) channel, which is expressed in nociceptors and responsible for the transport of positively charged ions, has been implicated in mediating nociceptive responses and pain perception [6,7]. Accordingly, antagonists of TRPA1 attracted attention as potential therapeutics for neuropathic pain. However, selective TRPA1 antagonists such as A-967079 and AMG0902 were not effective in attenuating mechanical allodynia induced by complete Freund's adjuvant or spinal nerve ligation [8]. These results indicate that the therapeutic effects of TRPA1 antagonists differ depending on the etiology of the insult causing the damage.

We have previously identified saikosaponins as TRPA1 antagonists and showed that saikosaponin D attenuated agonist-induced nociceptive responses and vincristine-induced mechanical hypersensitivity in mice [9]. Saikosaponins are phytochemicals consisting of glycan moieties and triterpenoid (aglycon or saikogenin) groups, and are mainly found in the genus Bupleurum, especially in Bupleurum falcatum[10]. Saikosaponins are integral to the pharmacological properties of $B$. falcatum in vitro and in vivo, including antiinflammation, immunomodulation, antitumor, and hepatoprotection [11]. In addition, saikosaponin A has been shown to alleviate mechanical hypersensitivity in an animal model of CCl-induced neuropathic pain [12]. Given these encouraging observations, the following studies were conducted to test the efficacy of saikosaponin $D$ in other neuropathic pain models, namely, diabetic neuropathic pain (DPN) and paclitaxel-induced mechanical hypersensitivity.

\section{Results}

While the mechanism remains unclear, accumulating evidence suggests the involvement of the TRPA1 channel in diabetes-induced neuropathic pain $[7,13]$. In order to test the effects of a TRPA1 antagonist, saikosaponin D ( $\vee$ Fig. 1), on neuropathic pain, we established a streptozotocin (STZ)-induced diabetic mouse model. Two intraperitoneal (i.p.) injections of STZ, given at 1-week intervals, led to significant increases in fasting blood glucose levels. At 4 weeks after the first i.p. injection of STZ, the fasting blood glucose levels in mice were as high as $400 \mathrm{mg} / \mathrm{dL}$. Mice with fasting blood glucose levels over $400 \mathrm{mg} / \mathrm{dL}$ were subject to the von Frey test to select the mice that showed mechanical hypersensitivity (paw withdrawal threshold $<0.2 \mathrm{~g}$ ). The paw withdrawal threshold for von Frey filament application in normal control mice at the same age was $1.12 \pm 0.21 \mathrm{~g}$. The selected mice were subjected to the von Frey test $1 \mathrm{~h}$ after oral administration of saikosaponin D (10 or $20 \mathrm{mg} / \mathrm{kg}, \mathrm{n}=7$

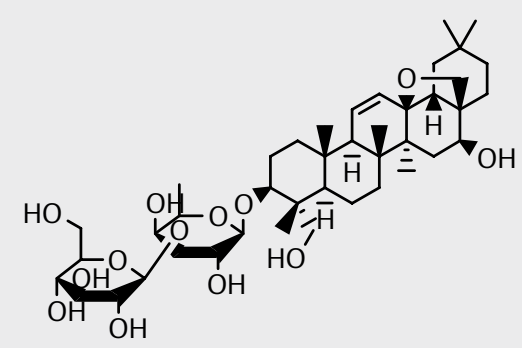

- Fig. 1 Chemical structure of saikosaponin D.

for each group) or a reference compound, pregabalin (30 mg/kg). The results showed that saikosaponin D significantly reversed STZinduced mechanical hypersensitivity. Saikosaponin D at a dose of $20 \mathrm{mg} / \mathrm{kg}$ improved the paw withdrawal threshold from $0.15 \pm 0.03$ to $0.54 \pm 0.13 \mathrm{~g}(\mathrm{p}<0.05)$ ( $>$ Fig. 2a). Pregabalin also improved the paw withdrawal threshold from $0.11 \pm 0.03$ to $0.84 \pm 0.21 \mathrm{~g}(p<0.01)$ ( Fig. 2a).

Five weeks after the first i.p. injection of STZ, the mice began to lose weight compared to the control mice (control $35.80 \pm 0.36$ vs. STZ 33.82 $\pm 0.73 \mathrm{~g} ; \mathrm{p}<0.05)$ and showed further increases in fasting blood glucose levels (control $129.1 \pm 4.4$ vs. STZ $448.2 \pm 24.8$ $\mathrm{mg} / \mathrm{dL}$ ) ( $\triangleright$ Fig. 2b). To further confirm the effects of saikosaponin $\mathrm{D}$, we repeated the von Frey test. Mice with fasting blood glucose levels over $400 \mathrm{mg} / \mathrm{dL}$ were subject to the von Frey test to select the mice that showed mechanical hypersensitivity. Then, separate groups of mice ( $n=7$ in each group) were orally administered saikosaponin D $(20 \mathrm{mg} / \mathrm{kg})$ or pregabalin $(30 \mathrm{mg} / \mathrm{kg}) 0.5$ or $2 \mathrm{~h}$ before the von Frey test. As shown in $\mathbf{F i g}$. $\mathbf{2 c}$, oral administration of saikosaponin D $0.5 \mathrm{~h}$ before the von Frey test resulted in a significant improvement of the paw withdrawal threshold when compared to STZ-treated mice (STZ only $0.11 \pm 0.03$ vs. STZ + saikosaponin D $0.93 \pm 0.18$; $p<0.001)$. Similarly, STZ-induced mechanical hypersensitivity was significantly attenuated by oral administration of saikosaponin D $2 \mathrm{~h}$ before the von Frey test (STZ only $0.11 \pm 0.03$ vs. STZ + saikosaponin D $0.67 \pm 0.15 ; \mathrm{p}<0.01$ ) ( $>$ Fig. 2 c). Oral administration of pregabalin $2 \mathrm{~h}$ before the von Frey test also improved the paw withdrawal threshold from $0.14 \pm 0.03$ to $1.25 \pm 0.27 \mathrm{~g}(\mathrm{p}<0.01)$ (> Fig. 2c).

Previous work done in our lab used the anticancer drug vincristine to establish a neuropathic pain animal model. Using this model, we observed that saikosaponin D significantly attenuated mechanical hypersensitivity in mice. Herein, we explored the utility of paclitaxel for use as an animal model of chemotherapy-induced peripheral neuropathy (CIPN). While i.p. injection of paclitaxel had little effect on weight gain compared to the control mice ( Fig. 3a), paclitaxel-induced mechanical hypersensitivity was evident on day 8 , and hypersensitivity persisted even after discontinuation of paclitaxel (days 15 and 22) as evidenced by the significantly reduced paw withdrawal thresholds to von Frey filaments when compared to the control group ( $\triangleright$ Fig. 3b). On day 8 , the paclitaxel-induced mechanical hypersensitivity was not attenuated by administration of saikosaponin D or a reference compound, gabapentin (data not shown). However, the repeated oral admin- 

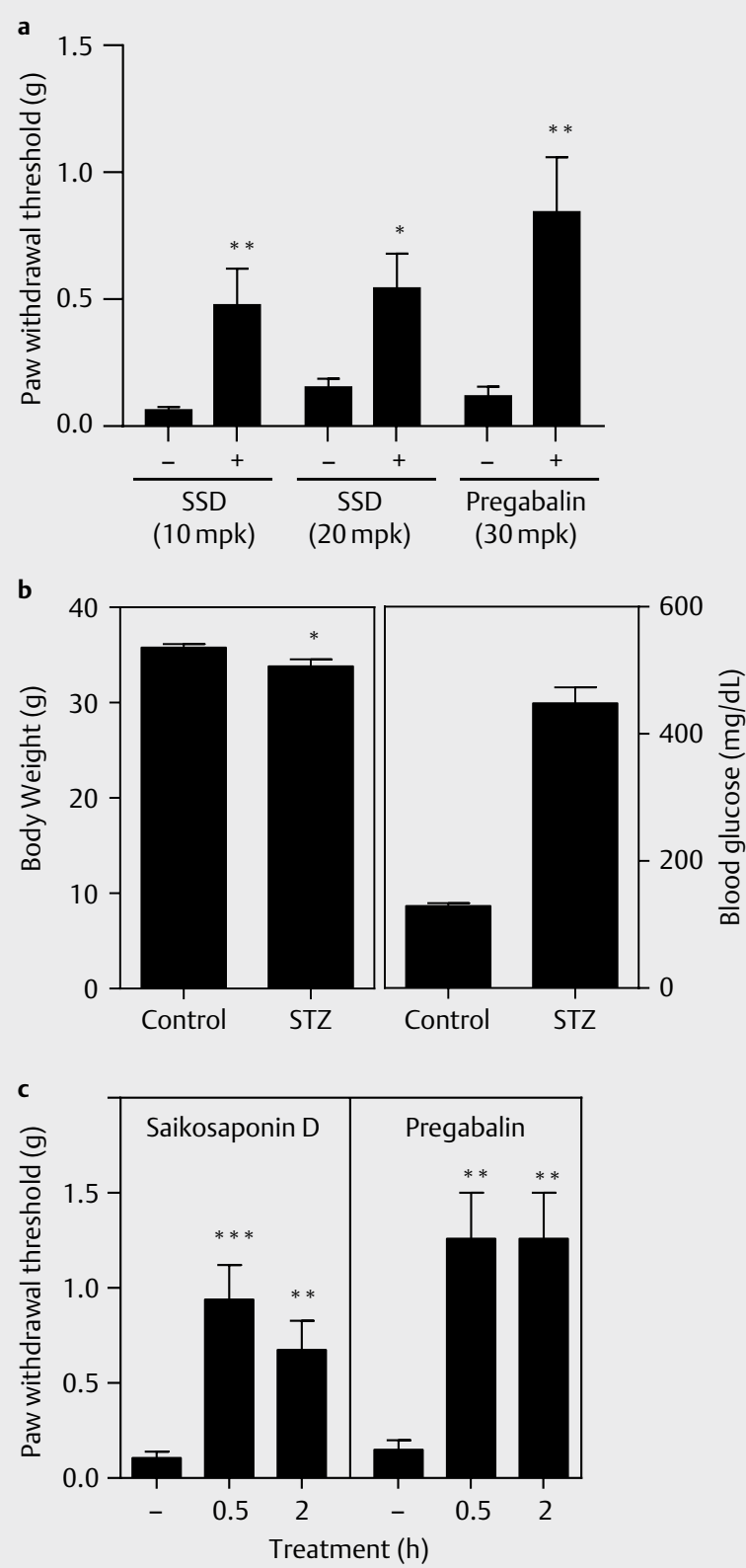

- Fig. 2 Effects of saikosaponin D on neuropathic pain in a mouse model of diabetes. a At 4 weeks after the first i.p. injection of STZ, the mice with fasting blood glucose levels over $400 \mathrm{mg} / \mathrm{dL}$ were subjected to the von Frey test before (-) and $1 \mathrm{~h}$ after (+) oral administration of either saikosaponin D or pregabalin. The paw withdrawal thresholds are expressed as the mean \pm SEM ( $n=7$ for each group). ${ }^{*} \mathrm{P}<0.01,{ }^{*} \mathrm{p}<0.05$, before (-) vs. $1 \mathrm{~h}$ after (+) saikosaponin $\mathrm{D}$ or pregabalin administration. SSD = saikosaponin D. $\mathbf{b}$ Body weight (left) and blood glucose level (right) were measured 5 weeks after the first i.p. injection of STZ. ${ }^{*} \mathrm{P}<0.05$, control vs. streptozotocin. c At 5 weeks after the first i.p. injection of STZ, the animals with fasting blood glucose levels over $400 \mathrm{mg} / \mathrm{dL}$ were subject to the von Frey test before (-) and 0.5 or $2 \mathrm{~h}$ after oral administration of saikosaponin D (20 mg/kg) or pregabalin $(30 \mathrm{mg} / \mathrm{kg})$. The paw withdrawal thresholds are expressed as the mean \pm SEM ( $n=7$ for each group). ${ }^{* * *} \mathrm{P}<0.001,{ }^{*}{ }^{*} \mathrm{P}<0.01$, before (-) vs. indicated time after saikosaponin D $(20 \mathrm{mg} / \mathrm{kg})$ or pregabalin $(30 \mathrm{mg} / \mathrm{kg})$ administration. a

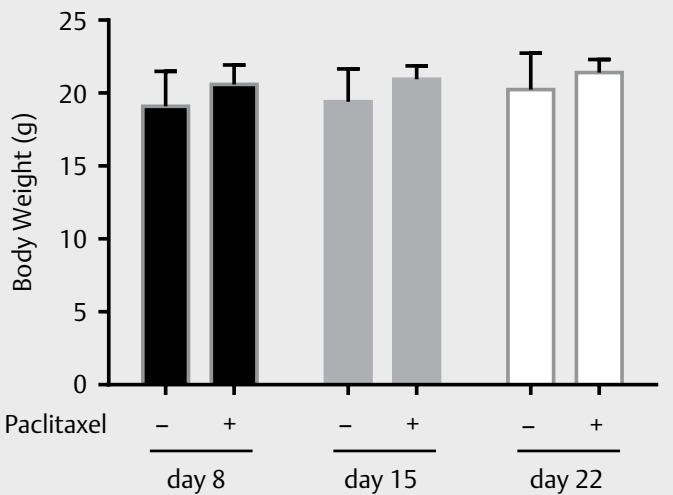

b
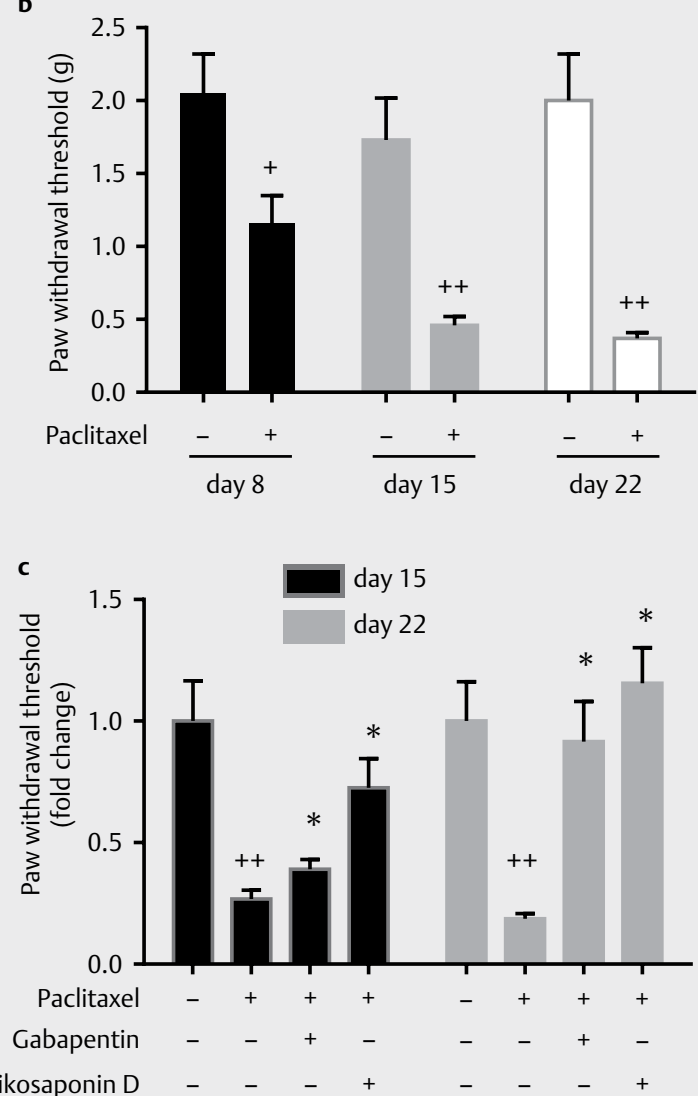

Fig. 3 Effects of saikosaponin D on mechanical hypersensitivity induced by paclitaxel. Separate groups of mice were left untreated or injected intraperitoneally with paclitaxel $(16 \mathrm{mg} / \mathrm{kg})$ three times per week for 2 weeks. Body weight a and paw withdrawal thresholds b were measured on days 8,15 , and $22 .+\mathrm{P}<0.05,{ }^{+}{ }^{+} \mathrm{p}<0.01$, control vs. paclitaxel administration. c Following induction of mechanical hypersensitivity, separate groups of mice were administered either a vehicle control, gabapentin $(10 \mathrm{mg} / \mathrm{kg}$, p.o.), or saikosaponin D (10 mg/kg, p.o.). Paw withdrawal thresholds were analyzed on days 15 and 22, and data are presented as the fold difference compared with the data for non-treated control mice (mean \pm SEM, $n=7) .+{ }^{+} P<0.01$, control vs. paclitaxel; ${ }^{*} \mathrm{p}<0.05$, paclitaxel vs. paclitaxel + gabapentin or saikosaponin D. 
istration of drugs and a von Frey evaluation on days 15 and 22 revealed that saikosaponin $\mathrm{D}$ and gabapentin indeed reversed paclitaxel-induced mechanical hypersensitivity. On day 22, both saikosaponin D and gabapentin improved the paw withdrawal thresholds to those seen in the control mice (control $100.0 \pm 16.2 \%$, saikosaponin D 115.5 $\pm 14.7 \%$, gabapentin $91.6 \pm 16.4 \%$; $<0.01$ ) ( $\triangleright$ Fig. $3 c$ ).

\section{Discussion}

Saikosaponins have been reported to exhibit a range of pharmacological activities both in vitro and in vivo[10]. Our group has previously reported the ability of saikosaponin $D$ to attenuate vincristine-induced mechanical hypersensitivity, and suggested that the activity of saikosaponin D is mediated, at least in part, through inhibition of the TRPA1 channel in nociceptors [9]. In the present study, other types of neuropathic pain animal models were used to gain more insight into the therapeutic effects of saikosaponin $D$.

Hiyama et al. [14] previously reported that the involvement of TRPA1 in STZ-induced mechanical hypersensitivity was not evident, because no difference was observed in paw withdrawal thresholds in the von Frey test between wild-type and Trpa $1 \%$ mice. In our study, it is not clear whether TRPA1 mediates mechanical hypersensitivity in STZ-induced diabetic animals and whether the activity of saikosaponin D is mediated through the inhibition of TRPA1. Nonetheless, we observed that saikosaponin D, when administered as early as $0.5 \mathrm{~h}$ before the von Frey test, significantly improved the paw withdrawal threshold in a STZ-induced neuropathic pain model ( Fig. 2c). These results strongly implicate the direct involvement of the molecular targets of saikosaponin D in STZ-induced mechanical hypersensitivity. Although the exact mechanism by which saikosaponin D exerts its activity in STZ-induced diabetic animals remains to be elucidated, we speculate that TRPA 1 antagonism by saikosaponin D contributes, at least in part, to improving paw withdrawal thresholds in STZ-induced diabetic animals.

CIPN is a painful adverse effect of anticancer drugs such as vincristine, bortezomib, oxaliplatin, and paclitaxel [15]. The involvement of TRPA1 in CIPN appears to differ depending on the type of anticancer drug. While Trpa $\%$ animals were less susceptible to vincristine-induced mechanical allodynia compared to wild-type mice [16], bortezomib-induced mechanical hypersensitivity was completely prevented by TRPA 1 deficiency [17]. In addition, paclitaxelinduced mechanical hypersensitivity was not affected by silencing of TRPA1-expressing fibers [18], indicating that TRPA1-expressing nociceptors have little effect on mediating paclitaxel-induced mechanical allodynia. In our study, 1-day oral administration of saikosaponin D had no effect on paclitaxel-induced mechanical allodynia; however, repeated administration of saikosaponin D significantly reduced paw withdrawal thresholds to von Frey filaments when compared to the control mice ( $\vee$ Fig. $\mathbf{3 c}$ ). These results suggest that the effect of saikosaponin $D$ is not attributed to the direct inhibition of the molecular targets that mediate paclitaxel-induced mechanical allodynia. Instead, given that saikosaponin D has a multitude of functions, it might be plausible to assume that the antiinflammatory activity of saikosaponin D mitigates oxidative stress caused by nerve damage and could account for improving paw withdrawal thresholds.
In conclusion, saikosaponin D improved mechanical hypersensitivity in animal models of DPN and CIPN. However, the mechanisms underlying the therapeutic effects of saikosaponin D appear to differ between STZ-induced and paclitaxel-induced pain models; STZ-induced mechanical hypersensitivity was alleviated $0.5 \mathrm{~h}$ after oral administration of saikosaponin D, while 1-day oral administration of saikosaponin D had little effect on the mechanical hypersensitivity induced by paclitaxel. Although the exact mechanism for the therapeutic effects of saikosaponin $D$ remains to be elucidated, our results shed new light on the therapeutic potential of saikosaponin $\mathrm{D}$ in the management of neuropathic pain caused by diabetes or chemotherapy.

\section{Materials and Methods}

\section{Chemicals}

Saikosaponin D (purity > $90 \%$ by HPLC), STZ (purity $>98 \%$ by HPLC), gabapentin (purity $>98 \%$ by HPLC), and pregabalin (purity $>95 \%$ by HPLC) were purchased from Merck. Paclitaxel (purity $>99 \%$ by HPLC) was purchased from TOCRIS.

\section{Mouse model for diabetic neuropathic pain}

Eight-week-old male ICR mice were purchased from Daehan Biolink and maintained in specific pathogen-free conditions for 1 week for acclimation. All animal experiments were approved by the Institutional Animal Care and Use Committee at Dankook University (No. DKU-19-028, September 9, 2019) and performed in accordance with the guidelines issued by the Korean Ministry of Food and Drug Safety and the NIH. Mice were injected intraperitoneally (i.p.) with STZ $(100 \mathrm{mg} / \mathrm{kg})$ twice at 1 -week intervals. To monitor blood glucose levels once a week, the mice were fasted for $6 \mathrm{~h}$ prior to obtaining blood samples via tail vein extraction. The concentration of glucose was measured using the Accu-Chek Performa blood glucose monitoring system. At weeks 4 or 5 after the first i.p. injection of STZ, the mice with fasting blood glucose levels over $400 \mathrm{mg} / \mathrm{dL}$ were used for the von Frey test and evaluation of saikosaponin D.

\section{Mouse model for paclitaxel-induced mechanical hypersensitivity}

Twelve-week-old female C57BL/6 mice were purchased from Samtaco and maintained in specific pathogen-free conditions for 1 week for acclimation. All animal experiments were approved by the Institutional Animal Care and Use Committee at Donga University (No. DIACUC-16-15, May 21, 2018) and performed in accordance with the guidelines issued by the Korean Ministry of Food and Drug Safety and the NIH. Mice were i.p. injected with paclitaxel (16 $\mathrm{mg} / \mathrm{kg}) 3$ times per week for 2 weeks and were orally administered vehicle, saikosaponin $D(10 \mathrm{mg} / \mathrm{kg})$, or gabapentin $(10 \mathrm{mg} / \mathrm{kg}$ ) daily from days 8 to 22. Paw withdrawal threshold to von Frey filaments was measured by two independent experimenters $1 \mathrm{~h}$ after administration of vehicle, saikosaponin $\mathrm{D}$, or gabapentin to assess the mechanical hypersensitivity on days 8, 15, and 22 .

\section{von Frey test}

The animals were placed on a mesh floor covered by a transparent glass dome and acclimated to the testing apparatus for $1 \mathrm{~h}$. 
von Frey filaments were applied to the plantar surface of the hind paw, and the paw withdrawal threshold was estimated by using a simplified up-down method as described previously [19].

\section{Statistical analysis}

Statistical significance was determined via one-way analysis of variance followed by the two-tailed Student's t-test performed in GraphPad Prism. P values of $<0.05$ were considered statistically significant.

\section{Funding}

This work was supported by the Support Program for Creative Industry Institutes (R0003950, MOTIE, Korea) and the National Research Foundation of Korea (NRF) grant funded by the Korea government (MIST) (NRF-2016R1A5A2007009 and NRF-2017R1C1B5014 853). We would also like to acknowledge the generous financial support from the Gyeonggi provincial government.

\section{Conflict of Interest}

The authors declare that they have no conflict of interest.

\section{References}

[1] Colloca L, Ludman T, Bouhassira D, Baron R, Dickenson AH, Yarnitsky D, Freeman R, Truini A, Attal N, Finnerup NB, Eccleston C, Kalso E, Bennett DL, Dworkin RH, Raja SN. Neuropathic pain. Nat Rev Dis Primers 2017; 3: 17002

[2] Fallon MT. Neuropathic pain in cancer. Br ] Anaesth 2013; 111: 105-111

[3] Basbaum AI, Bautista DM, Scherrer G, Julius D. Cellular and molecular mechanisms of pain. Cell 2009; 139: 267-284

[4] St John Smith E. Advances in understanding nociception and neuropathic pain. J Neurol 2018; 265: 231-238

[5] Campbell JN, Meyer RA. Mechanisms of neuropathic pain. Neuron 2006; 52: 77-92

[6] Nativi C, Gualdani R, Dragoni E, Di Cesare Mannelli L, Sostegni S, Norcini M, Gabrielli G, la Marca G, Richichi B, Francesconi O, Moncelli MR, Ghelardini C, Roelens S. A TRPA1 antagonist reverts oxaliplatininduced neuropathic pain. Sci Rep 2013; 3: 2005

[7] Wang S, Kobayashi K, Kogure Y, Yamanaka H, Yamamoto S, Yagi H, Noguchi K, Dai Y. Negative regulation of TRPA1 by AMPK in primary sensory neurons as a potential mechanism of painful diabetic neuropathy. Diabetes 2018; 67: 98-109
[8] Lehto SG, Weyer AD, Youngblood BD, Zhang M, Yin R, Wang W, Teffera Y, Cooke M, Stucky CL, Schenkel L, Geuns-Meyer S, Moyer BD, Wild KD, Gavva NR. Selective antagonism of TRPA1 produces limited efficacy in models of inflammatory- and neuropathic-induced mechanical hypersensitivity in rats. Mol Pain 2016; 12: 1744806916677761

[9] Lee G, Choi J, Nam Y], Song MJ, Kim JK, Kim WJ, Kim P, Lee JS, Kim S, No KT, Lee JH, Lee JK, Choi Y. Identification and characterization of saikosaponins as antagonists of transient receptor potential A1 channel. Phyto Res 2020; 34: 788-795

[10] Ashour ML, Wink M. Genus Bupleurum: a review of its phytochemistry, pharmacology and modes of action. J Pharm Pharmacol 2011; 63: 305-321

[11] Yuan B, Yang R, Ma Y, Zhou S, Zhang X, Liu Y. A systematic review of the active saikosaponins and extracts isolated from Radix Bupleuri and their applications. Pharm Biol 2017; 55: 620-635

[12] Zhou X, Cheng H, Xu D, Yin Q, Cheng L, Wang L, Song S, Zhang M. Attenuation of neuropathic pain by saikosaponin a in a rat model of chronic constriction injury. Neurochem Res 2014; 39: 2136-2142

[13] Barriere DA, Rieusset J, Chanteranne D, Busserolles J, Chauvin MA, Chapuis L, Salles J, Dubray C, Morio B. Paclitaxel therapy potentiates cold hyperalgesia in streptozotocin-induced diabetic rats through enhanced mitochondrial reactive oxygen species production and TRPA1 sensitization. Pain 2012; 153: 553-561

[14] Hiyama H, Yano Y, So K, Imai S, Nagayasu K, Shirakawa H, Nakagawa T, Kaneko S. TRPA1 sensitization during diabetic vascular impairment contributes to cold hypersensitivity in a mouse model of painful diabetic peripheral neuropathy. Mol Pain 2018; 14: 1744806918789812

[15] Starobova H, Vetter I. Pathophysiology of Chemotherapy-Induced Peripheral Neuropathy. Front Mol Neurosci 2017; 10: 174

[16] Old EA, Nadkarni S, Grist J, Gentry C, Bevan S, Kim KW, Mogg A], Perretti M, Malcangio M. Monocytes expressing CX3CR1 orchestrate the development of vincristine-induced pain. J Clin Invest 2014; 124: 2023-2036

[17] Trevisan G, Materazzi S, Fusi C, Altomare A, Aldini G, Lodovici M, Patacchini R, Geppetti P, Nassini R. Novel therapeutic strategy to prevent chemotherapy-induced persistent sensory neuropathy by TRPA 1 blockade. Cancer Res 2013; 73: 3120-3131

[18] Shim HS, Bae C, Wang J, Lee KH, Hankerd KM, Kim HK, Chung JM, La $J \mathrm{H}$. Peripheral and central oxidative stress in chemotherapy-induced neuropathic pain. Mol Pain 2019; 15: 1744806919840098

[19] Bonin RP, Bories C, De Koninck Y. A simplified up-down method (SUDO) for measuring mechanical nociception in rodents using von Frey filaments. Mol Pain 2014; 10: 26 